\title{
Prevalence of Depression during Pregnancy in Spanish Women: Trajectory and Risk Factors in Each Trimester
}

\author{
M. Carmen Míguez * and M. Belén Vázquez
}

check for updates

Citation: Míguez, M.C.; Vázquez, M.B. Prevalence of Depression during Pregnancy in Spanish Women: Trajectory and Risk Factors in Each Trimester. Int. J. Environ. Res. Public Health 2021, 18, 6789. https:// doi.org/10.3390/ijerph18136789

Academic Editor: Paul B. Tchounwou

Received: 20 May 2021

Accepted: 21 June 2021

Published: 24 June 2021

Publisher's Note: MDPI stays neutral with regard to jurisdictional claims in published maps and institutional affiliations.

Copyright: (c) 2021 by the authors. Licensee MDPI, Basel, Switzerland. This article is an open access article distributed under the terms and conditions of the Creative Commons Attribution (CC BY) license (https:// creativecommons.org/licenses/by/ $4.0 /)$.
Department of Clinical Psychology and Psychobiology, Faculty of Psychology, University of Santiago de Compostela, 15782 Santiago de Compostela, Spain; mariabelen.vazquez.gomez1@rai.usc.es

* Correspondence: mcarmen.miguez@usc.es; Tel.: +34-881-81-3740

\begin{abstract}
The aims of this research were to determine the trajectories of probable depression and major depression during pregnancy and to identify the associated and predictor variables (sociodemographic, pregnancy-related, and psychological) for both conditions in each trimester of pregnancy. A longitudinal study was carried out with 569 pregnant Spanish women who were assessed in the first, second, and third trimesters of pregnancy. Depression was assessed using the Edinburgh Postnatal Depression Scale and a clinical interview. Measures of anxiety and stress were also included. The prevalence of probable depression in the first, second, and third trimesters was $23.4 \%$, $17.0 \%$, and $21.4 \%$, respectively, and that of major depression was $5.1 \%, 4.0 \%$, and $4.7 \%$. Thus, the prevalence of both conditions was the highest in the first and third trimesters. The trajectories of probable depression and major depression followed the same pattern throughout pregnancy. All of the psychological variables studied were associated with both conditions in all three trimesters, with perceived stress being a predictor at all times. The association between the other variables and both conditions of depression was similar. Two exceptions stand out: having had previous miscarriages, which was only associated with probable depression and was also a predictor, in the first trimester; and complications during pregnancy, which was only associated with probable and major depression in the third trimester. These findings should be taken into account in routine pregnancy follow-ups, and necessary interventions should be started in the first trimester.
\end{abstract}

Keywords: pregnancy; antenatal depression; prevalence; trajectory; risk factors

\section{Introduction}

Depression is one of the most prevalent psychological disorders, affecting women at twice the rate of men [1]. In women, vulnerability to depression is particularly high during pregnancy and in the postpartum period [2], as the birth of a child is a life event associated with numerous biological, hormonal, psychological, familial, and social changes.

Antenatal depression has received less attention than postpartum depression [3], as pregnancy was traditionally thought to protect women against the onset or relapse of depressive disorders. Depression during pregnancy has important consequences, both for mothers and their children. In particular, the main consequence of antenatal depression for mothers is a continuation of the state into the postpartum period, as depression in pregnancy is the main risk factor for postpartum depression [4-6]. Children of mothers with antenatal depression have been found to be more likely to have intrauterine growth retardation [7], be born preterm [7-10], and be small for gestational age [9,10], all of which are major causes of neonatal and infant morbidity and mortality.

On the other hand, there is a lack of longitudinal research addressing the prevalence of antenatal depression in each trimester of pregnancy, as most studies are cross-sectional, which makes it impossible to determine the trajectory of depression throughout pregnancy. This can lead to an inaccurate picture of the actual situation, as the prevalence of depression often varies throughout pregnancy [11-13]. The analysis of the trajectory of depression in the same sample of pregnant women is important because it enables the identification of 
the times of the greatest vulnerability since cross-sectional studies provide very different results, as they use different assessment instruments.

The few existing longitudinal studies have provided mixed results regarding the trajectory of depression. In particular, some studies found that the first and third trimesters of pregnancy are the times of the highest prevalence of probable depression [13-16] and major depression [13], although the values in the third trimester did not reach those observed in the first trimester. However, in a study conducted in China [17], although probable depression was also the most prevalent in the first and third trimesters, the prevalence rate was higher in the latter than in the former. The same pattern was observed in Italy for major depression [18]. In all cases, the trajectory of depression is V-shaped. By contrast, a study in India [19] reported the opposite trajectory $(\bigwedge)$, with the highest prevalence occurring in the second trimester and the lowest prevalence in the third trimester. On the other hand, a study conducted in Korea [20] found that the presence of probable depression followed an ascending path (/) between the first and the third trimester.

As longitudinal studies on the prevalence of antenatal depression by trimester are scarce, there is also a lack of knowledge regarding the associated variables at each point. Specifically, we did not find any studies in the existing literature that have analysed the variables associated with antenatal depression in each trimester. Thus, it is not possible to know whether there are variables associated with depression that remain stable throughout the pregnancy, or whether there are others that are specific to a particular trimester and then disappear in the rest of the pregnancy. Identifying such associations would enable the implementation of prevention and intervention measures adjusted to each phase. In this respect, the variables that are the most frequently associated with depression in pregnancy are sociodemographic, obstetric, and psychological variables [21]. In particular, antenatal depression has been associated with low educational level [15,22-25] and low socio-economic level [17,22,24,26-29], being unemployed [22,30,31], and not having planned the pregnancy $[15,17,19,25,27,29-34]$. Regarding psychological variables, having a history of depression $[17,23,26,31]$, as well as anxiety $[26,33-36]$ or stress $[33,34,36]$ are the variables that are the most frequently associated with antenatal depression. On the other hand, in regard to variables such as age and parity, the studies' findings are contradictory. Specifically, different studies have reported a relationship with younger age [18,37], while in others, it is related to older age $[23,29]$. Likewise, with respect to parity, both primiparity [36] and multiparity $[19,23,26,31,32]$ have been associated with the presence of antenatal depression.

Monitoring women for depression throughout pregnancy will enable the identification of the most vulnerable phases and the variables associated with the presence of depression in each trimester. This would lead to more efficient help being offered in accordance with the real needs of future mothers and will prevent the depressive state and its associated consequences from extending to the postpartum period.

Therefore, the aims of the present research were first, to assess the trajectory of probable depression, assessed with a self-report instrument (EPDS), and of major depression, assessed with a clinical interview (SCID), during pregnancy; and second, to identify the associated and predictor variables (sociodemographic, pregnancy-related, and psychological) of both probable depression and major depression in each trimester of pregnancy. The study aimed to answer the following specific questions:

1. Is the trajectory of depression throughout pregnancy the same regardless of its severity?

2. Do the same variables predict the presence of probable depression and of major depression?

3. Which variables are the most closely associated with depression in each trimester? Are they the same, or are there trimester-specific predictors?

\section{Materials and Methods}

\subsection{Procedure and Participants}

The present research was conducted in accordance with the Helsinki Declaration and received previous approval from the ethics committees of all of the institutions involved. 
It was a longitudinal study with three assessment waves: the 1st trimester of pregnancy $(\mathrm{M}=10.87$ weeks; $\mathrm{SD}=2.36)$, the 2 nd trimester of pregnancy $(\mathrm{M}=20.69$ weeks; $\mathrm{SD}=1.21)$ and the 3rd trimester of pregnancy $(M=33.28$ weeks; $S D=2.05)$. Pregnant women attending the primary public healthcare service in northwest Spain were recruited in the 1 st trimester of pregnancy $(n=620)$. Women were eligible to participate if they were at least 18 years of age, were in the first trimester of pregnancy, spoke Spanish, and were willing to participate in subsequent assessments throughout the pregnancy. The exclusion criteria were: being under 18 years of age, having a gestational age equal to or greater than 15 weeks, not reading or speaking Spanish, and not having participated in any of the evaluations. The aims and procedures were explained, and the pregnant women who were willing to participate provided written informed consent. The final sample consisted of 569 women. The procedure and sample tracking characteristics are shown in Figure 1.

All women participated voluntarily in the study. The assessments were carried out personally and individually at the hospital prior to the women entering the protocolised pregnancy follow-up consultation. In each pregnancy trimester, all of the questionnaires and SCID interviews were administered personally by one psychologist who received SCID training and who was blind to the EPDS scores. The average duration of each interview was about $40 \mathrm{~min}$.

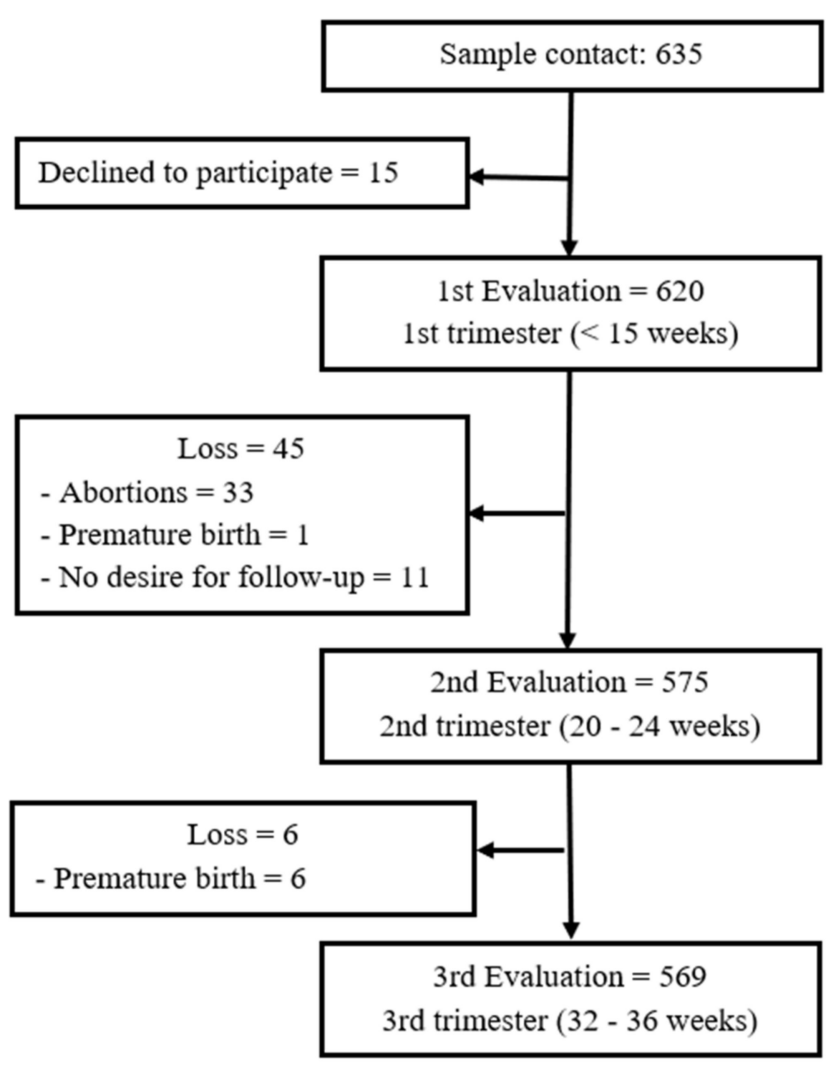

Figure 1. Flow diagram of recruitment and progress through the study.

\subsection{Measures}

\subsubsection{Socio-Demographic and Pregnancy Information}

Three ad hoc questionnaires including questions on socio-demographic (e.g., age, marital status, educational level, occupational status, and personal monthly income), pregnancy-related (e.g., parity, previous abortions, planned pregnancy, pregnancy reaction, complications, attendance at maternal classes), and psychological variables were elaborated on specifically for the study. 


\subsubsection{Depression}

The Edinburgh Postnatal Depression Scale (EPDS) [38] is a self-reported questionnaire consisting of 10 items with 4 response options. The scores range between 0 and 30, with higher scores indicating a greater severity of depression. The Spanish validation of the EPDS for use in pregnancy was used [13], which determined that the most appropriate cut-off point for screening for probable antenatal depression was $\geq 10$. The reliability of the EPDS during pregnancy was 0.81 in the first trimester, 0.82 in the second trimester, and 0.85 in the third trimester in the present study.

The Structured Clinical Interview for DSM-IV (SCID) [39] is a semi-structured interview that determines a formal diagnosis according to the Diagnostic and Statistical Manual of Mental Disorders (DSM-IV). The use of such interviews improves diagnostic reliability by standardising the assessment process and increases the diagnostic validity by facilitating the application of DSM diagnostic criteria and the systematic enquiry of symptoms that might otherwise go unnoticed.

\subsubsection{Stress}

The Spanish validation [40] of the Perceived Stress Scale (PSS) [41] was used. The PSS is a self-administered scale that measures the degree to which life situations in the previous month are rated as stressful. It consists of 14 items, with 5 response options. The scale ranges from 0 to 56 , with higher scores indicating a higher level of perceived stress. With this sample, the reliability of the PSS during pregnancy was 0.86 in the first trimester, 0.88 in the second trimester, and 0.87 in the third trimester.

\subsubsection{Anxiety}

The State-Trait Anxiety Inventory (STAI) [42] assesses both the current level of anxiety and the individual's predisposition to suffering from anxiety. It consists of 40 items, 20 of which refer to the state subscale (STAI-E), with other 20 referring to the trait subscale (STAI-R). The score for each subscale ranges from 0 to 60 , with higher scores indicating higher levels of anxiety. For the state subscale, the recommended cut-off point for women is greater than 31, and for trait anxiety, the cut-off point for woman is greater than 32 (75th percentile). In our sample, the reliability of the trait anxiety subscale in the first trimester of pregnancy was 0.88 , and the reliability of the state subscale, 0.91 . In the second and third trimesters, the reliability of the state anxiety subscale was 0.92 .

\subsection{Data Analysis}

Data were analyzed using SPSS Statistics, version 22 (PASW Statistics for Windows, SPSS Inc., Chicago, IL, USA), and a significance level of $p<0.05$ was applied. To test the differences between the presence or absence of depression, a chi-square test for discrete variables and Student's t-tests for continuous variables were performed. Cramers's V coefficients and Cohen's d were calculated in order to estimate the size of the effect.

Binary forward stepwise logistic regression analysis was also used to determine the variables predicting depression in pregnancy, according to the EPDS, and/or major depression, according to the SCID, in each trimester of pregnancy. The dependent variable was probable depression status (EPDS $\geq 10$, yes/EPDS $<10$, no) or major depression status (yes depression/no depression), and the independent variables were those variables for which significant differences were found in the two-by-two analyses. Likewise, Cronbach's alpha $(\alpha)$ was calculated in order to estimate the reliability of the scales.

\section{Results}

\subsection{Characteristics of the Sample}

The study sample was composed of 569 women ranging in age from 18 to 45 years, with a mean age of 32.80 years $(\mathrm{SD}=4.75)$. Most of the women were married or cohabiting $(94.9 \%) ; 46.4 \%(n=264)$ had university education, and 35.3\% $(n=201)$ had secondary education. Regarding employment status, $75.2 \%(n=428)$ were working. Regarding 
personal monthly income, $44.6 \%(n=254)$ stated that they earned less than 1000 euros per month. Pregnancy was planned in $85.9 \%(n=489)$ of cases, $59.4 \%(n=338)$ of the women were primiparous, and $93.0 \%(n=529)$ reported having reacted positively to confirmation of the pregnancy. Likewise, $63.3 \%(n=360)$ reported attending maternal education classes, with the mean attendance being 4.45 classes $(S D=4.33)$ out of the 10 classes offered on a regular basis.

\subsection{Trajectory of Depression throughout Pregnancy}

The prevalence of depression varied according to the time of the assessment and the assessment instrument used (Figure 2). The first trimester was the period during which the highest percentage of women had probable depression $(23.4 \%)$ or major depression $(5.1 \%)$. The prevalence of both probable depression $(17.0 \%)$ and major depression $(4.0 \%)$ was the lowest in the second trimester. In the third trimester, the prevalence of both probable depression $(21.4 \%)$ and major depression $(4.7 \%)$ was higher than in the second trimester but did not reach the values observed in the first trimester. Thus, the trajectories of probable depression and major depression followed the same pattern throughout pregnancy.

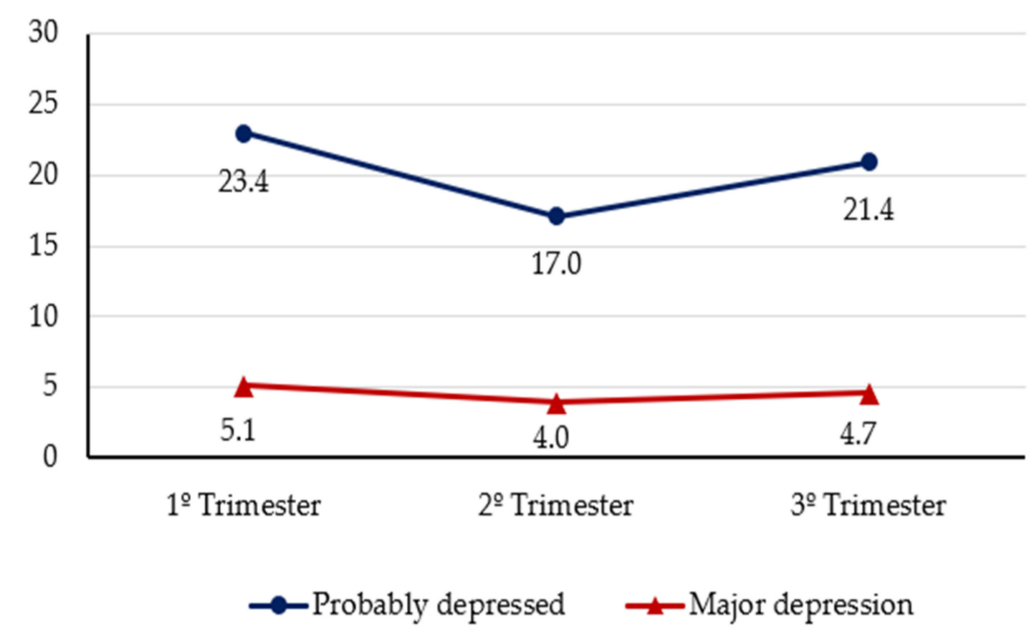

Figure 2. Trajectory of the prevalence of probable depression and major depression during pregnancy.

\subsection{Variables Associated with the Presence of Depression}

\subsubsection{Sociodemographic Variables}

Regarding the sociodemographic variables, the characteristics of the pregnant women experiencing probable depression or major depression in each trimester of pregnancy as well as the variables associated with both types of depression are shown in Table 1. Regarding the mean age, significant differences were found in the prevalence of probable depression in the first (31.99 vs. 33.05; $\mathrm{t}(569)=2.254, p=0.025, \mathrm{~d}$ Cohen $=0.25)$ and second trimesters (31.63 vs. $33.04 ; \mathrm{t}(569)=2.686, p=0.007$, $\mathrm{d}$ Cohen $=0.28)$, as these women were younger than the women who did not experience probable depression. For women experiencing major depression, the mean age was statistically significantly lower in the second $(\mathrm{t}(569)=4.158, p<0.001, \mathrm{~d}$ Cohen $=0.82 ; 28.83$ vs. 32.97) and third trimesters $(\mathrm{t}(569)$ $=4.768, p<0.001$, $\mathrm{d}$ Cohen $=0.86 ; 28.63$ vs. 33.01 ).

Having a lower level of education was associated with both depression conditions in all three trimesters of pregnancy, while a lower level of income was only associated with probable depression in the 1st and 2nd trimesters. On the other hand, not having a partner, not cohabiting, or not being married was not associated with either condition at any time.

\subsubsection{Pregnancy-Related Variables}

In terms of pregnancy-related variables, the characteristics of pregnant women with probable depression and major depression in each trimester of pregnancy as well as the variables associated with both conditions are shown in Table 2. 
Having reacted negatively to the confirmation of pregnancy was associated with both probable and major depression at all time points. On the other hand, having had previous miscarriages was only associated with probable depression in the first trimester, while having had complications at some time during pregnancy was only associated with the presence of major depression in the third trimester.

\subsubsection{Psychological Variables}

Regarding the psychological variables, the characteristics of pregnant women with probable depression and major depression in each trimester of pregnancy as well as the variables associated with both types of depression are shown in Table 3.

Regarding perceived stress, significantly higher mean scores were found among women experiencing probable depression and women experiencing major depression. Specifically, women with probable depression had higher mean scores for perceived stress in the first $(\mathrm{t}(569)=-14.332, p<0.001, \mathrm{~d}$ Cohen $=-1.63 ; 25.44$ vs. 16.17), second $(\mathrm{t}(569)=$ $-15.11, p<0.001$, $\mathrm{d}$ Cohen $=-1.81 ; 25.69$ vs. 14.55$)$ and third trimesters $(\mathrm{t}(569)=-14.946$, $p<0.001, \mathrm{~d}$ Cohen $=-1.59 ; 25.33$ vs. 15.08$)$. In addition, the mean scores were significantly higher among women with major depression in the first $(\mathrm{t}(569)=-6.234, p<0.001, \mathrm{~d}$ Cohen $=-1.30 ; 26.66$ vs. 17.89), second $(\mathrm{t}(569)=-7.953, p<0.001$, $\mathrm{d}$ Cohen $=-1.82 ; 28.52$ vs. 15.94$)$ and third trimesters $(\mathrm{t}(569)=-8.343, p<0.001$, $\mathrm{d}$ Cohen $=-1.74 ; 29.00$ vs. 16.69$)$.

All of the psychological variables studied were associated with both probable and major depression at all times. Thus, having a prior history of depression, having experienced a worsening of mood in previous pregnancies, having elevated state and trait anxiety as well as a higher level of stress were significantly associated with both probable depression and major depression.

Table 1. Characteristics of women with probable depression and major depression as a function of sociodemographic variables.

\begin{tabular}{|c|c|c|c|c|c|c|c|c|c|c|c|c|}
\hline \multirow{3}{*}{ First Trimester } & \multicolumn{6}{|c|}{ Probable Depression } & \multicolumn{6}{|c|}{ Major Depression } \\
\hline & \multicolumn{2}{|c|}{$\begin{array}{c}\text { No } \\
(n=436)\end{array}$} & \multicolumn{2}{|c|}{$\begin{array}{c}\text { Yes } \\
(n=133)\end{array}$} & \multirow[b]{2}{*}{$\chi^{2}$} & \multirow[b]{2}{*}{$\mathrm{V}$} & \multicolumn{2}{|c|}{$\begin{array}{c}\text { No } \\
(n=540)\end{array}$} & \multicolumn{2}{|c|}{$\begin{array}{c}\text { Yes } \\
(n=29)\end{array}$} & \multirow[b]{2}{*}{$\chi^{2}$} & \multirow[b]{2}{*}{$\mathrm{V}$} \\
\hline & $n$ & $\%$ & $n$ & $\%$ & & & $n$ & $\%$ & $n$ & $\%$ & & \\
\hline \multicolumn{13}{|l|}{ Age } \\
\hline$\leq 30$ & 121 & 27.8 & 55 & 41.4 & 8.825 & 0.13 & 166 & 30.7 & 10 & 34.5 & 0.180 & \\
\hline$>30$ & 315 & 72.2 & 78 & 58.6 & & & 374 & 69.3 & 19 & 65.5 & & \\
\hline \multicolumn{13}{|l|}{ Marital Status } \\
\hline Unmarried & 20 & 4.6 & 9 & 6.8 & 1.001 & & 25 & 4.6 & 4 & 13.8 & 4.778 & \\
\hline Married/Cohabiting & 416 & 95.4 & 124 & 93.2 & & & 515 & 95.4 & 25 & 86.2 & & \\
\hline Level of Education & & & & & & & & & & & & \\
\hline Primary/Secondary & 215 & 49.3 & 90 & 67.7 & 13.809 & 0.16 & 284 & 52.6 & 21 & 72.4 & 4.348 & 0.09 \\
\hline University & 221 & 50.7 & 43 & 32.3 & & & 256 & 47.4 & 8 & 27.6 & & \\
\hline \multicolumn{13}{|l|}{ Employment Status } \\
\hline Working & 339 & 77.8 & 89 & 66.9 & $\begin{array}{l}6.419 \\
*\end{array}$ & 0.11 & 410 & 75.9 & 18 & 62.1 & 2.835 & \\
\hline Not Working & 97 & 22.2 & 44 & 33.1 & & & 130 & 24.1 & 11 & 37.9 & & \\
\hline \multicolumn{13}{|l|}{ Pregnancy Influenced } \\
\hline Employment & & & & & 14.979 & & & & & & 8.892 & \\
\hline No & 365 & 83.7 & 91 & 68.4 & $* * *$ & 0.16 & 439 & 81.3 & 17 & 58.6 & $* *$ & 0.13 \\
\hline Yes & 71 & 16.3 & 42 & 31.6 & & & 101 & 18.7 & 12 & 41.4 & & \\
\hline \multicolumn{13}{|l|}{ Monthly Income (Euros) } \\
\hline$\leq 1000$ & 183 & 48.2 & 71 & 65.1 & $\begin{array}{c}9.783 \\
* *\end{array}$ & 0.14 & 240 & 51.6 & 14 & 58.3 & 0.413 & \\
\hline$>1000$ & 197 & 51.8 & 38 & 34.9 & & & 225 & 48.4 & 10 & 41.7 & & \\
\hline Second Trimester & \multicolumn{2}{|c|}{$\begin{array}{c}\text { No } \\
(n=472)\end{array}$} & \multicolumn{2}{|c|}{$\begin{array}{c}\text { Yes } \\
(n=97)\end{array}$} & & & \multicolumn{2}{|c|}{$\begin{array}{c}\text { No } \\
(n=546)\end{array}$} & \multicolumn{2}{|c|}{$\begin{array}{c}\text { Yes } \\
(n=23)\end{array}$} & & \\
\hline Age & & & & & & & & & & & & \\
\hline$\leq 30$ & 134 & 28.4 & 42 & 43.3 & $\begin{array}{c}8.3 / 2 \\
* *\end{array}$ & 0.12 & 159 & 29.1 & 17 & 73.9 & ${ }_{* * *}^{20.127}$ & 0.19 \\
\hline$>30$ & 338 & 71.6 & 55 & 56.7 & & & 387 & 70.9 & 6 & 26.1 & & \\
\hline
\end{tabular}


Table 1. Cont.

\begin{tabular}{|c|c|c|c|c|c|c|c|c|c|c|c|c|}
\hline Marital Status & & & & & & & & & & & & \\
\hline Unmarried & 23 & 4.9 & 6 & 6.2 & \multirow[t]{2}{*}{0.287} & & 27 & 4.9 & 2 & 8.7 & \multicolumn{2}{|l|}{0.642} \\
\hline Married/Cohabiting & 449 & 95.1 & 91 & 93.8 & & & 519 & 95.1 & 21 & 91.3 & & \\
\hline Level of Education & & & & & \multirow{3}{*}{$\underset{* * *}{16.200}$} & \multirow{3}{*}{0.17} & & & & & \multirow{3}{*}{$\underset{* *}{8.108}$} & \multirow{3}{*}{0.12} \\
\hline Primary/Secondary & 235 & 49.8 & 70 & 72.2 & & & 286 & 52.4 & 19 & 82.6 & & \\
\hline University & 237 & 50.2 & 27 & 27.8 & & & 260 & 47.6 & 4 & 17.4 & & \\
\hline Employment Status & & & & & \multirow{3}{*}{1.642} & & & & & & \multirow{3}{*}{$\underset{* * *}{12.956}$} & \multirow{3}{*}{0.15} \\
\hline Working & 360 & 76.3 & 68 & 70.1 & & & 418 & 76.6 & 10 & 43.5 & & \\
\hline Not Working & 112 & 23.7 & 29 & 29.9 & & & 128 & 23.4 & 13 & 56.5 & & \\
\hline Pregnancy Influenced & & & & & \multirow{4}{*}{$\underset{* * *}{24.166}$} & & & & & & & \multirow{4}{*}{0.14} \\
\hline Employment & & & & & & & & & & & \multirow{3}{*}{$\underset{* *}{11.231}$} & \\
\hline No & 367 & 77.8 & 52 & 53.6 & & 0.21 & 409 & 74.9 & 10 & 43.5 & & \\
\hline Yes & 105 & 22.2 & 45 & 46.4 & & & 137 & 25.1 & 13 & 56.5 & & \\
\hline Monthly Income (euros) & & & & & \multirow{3}{*}{$\underset{* *}{7.442}$} & \multirow{3}{*}{0.12} & & & & & \multirow{3}{*}{1.872} & \\
\hline$\leq 1000$ & 199 & 49.1 & 55 & 65.5 & & & 243 & 51.4 & 11 & 68.8 & & \\
\hline$>1000$ & 206 & 50.9 & 29 & 34.5 & & & 230 & 48.6 & 5 & 31.3 & & \\
\hline Third trimester & \multicolumn{2}{|c|}{$\begin{array}{c}\text { No } \\
(n=447)\end{array}$} & \multicolumn{2}{|c|}{$\begin{array}{c}\text { Yes } \\
(n=122)\end{array}$} & & & \multicolumn{2}{|c|}{$\begin{array}{c}\text { No } \\
(n=542)\end{array}$} & \multicolumn{2}{|c|}{$\begin{array}{c}\text { Yes } \\
(n=27)\end{array}$} & & \\
\hline Age & & & & & \multirow{3}{*}{3.335} & & & & & & \multirow{3}{*}{$\underset{* * *}{20.637}$} & \multirow{3}{*}{0.19} \\
\hline$\leq 30$ & 130 & 29.1 & 46 & 37.7 & & & 157 & 29.0 & 19 & 70.4 & & \\
\hline$>30$ & 317 & 70.9 & 76 & 62.3 & & & 385 & 71.0 & 8 & 29.6 & & \\
\hline Marital Status & & & & & & & & & & & & \\
\hline Unmarried & 22 & 4.9 & 7 & 5.7 & \multirow[t]{2}{*}{0.132} & & 27 & 5.0 & 2 & 7.4 & \multirow[t]{2}{*}{0.313} & \\
\hline Married/Cohabiting & 425 & 95.1 & 115 & 94.3 & & & 515 & 95.0 & 25 & 92.6 & & \\
\hline Level of Education & & & & & \multirow{3}{*}{$\begin{array}{c}8.948 \\
* *\end{array}$} & & & & & & & \\
\hline Primary/Secondary & 225 & 50.3 & 80 & 65.6 & & 0.13 & 284 & 52.4 & 21 & 77.8 & $\begin{array}{c}6.661 \\
*\end{array}$ & 0.11 \\
\hline University & 222 & 49.7 & 42 & 34.4 & & & 258 & 47.6 & 6 & 22.2 & & \\
\hline Employment Status & & & & & & & & & & & & \\
\hline Working & 344 & 77.0 & 84 & 68.9 & 3.378 & & 415 & 76.6 & 13 & 48.1 & 11.145 & 0.14 \\
\hline Not Working & 103 & 23.0 & 38 & 31.1 & & & 127 & 23.4 & 14 & 51.9 & & \\
\hline Pregnancy Influenced & & & & & & & & & & & & \\
\hline Employment & & & & & 4.066 & & & & & & & \\
\hline No & 290 & 64.9 & 67 & 54.9 & $*$ & 0.09 & 342 & 63.1 & 15 & 55.6 & 0.626 & \\
\hline Yes & 157 & 35.1 & 55 & 45.1 & & & 200 & 36.9 & 12 & 44.4 & & \\
\hline Monthly Income (euros) & & & & & 1.798 & & & & & & & \\
\hline$\leq 1000$ & 195 & 50.4 & 59 & 57.8 & & & 242 & 51.5 & 12 & 63.2 & 0.996 & \\
\hline$>1000$ & 192 & 49.6 & 43 & 42.2 & & & 228 & 48.5 & 7 & 36.8 & & \\
\hline
\end{tabular}

Table 2. Characteristics of women with probable depression and major depression as a function of pregnancy-related variables.

\begin{tabular}{|c|c|c|c|c|c|c|c|c|c|c|c|c|}
\hline \multirow{3}{*}{ First Trimester } & \multicolumn{6}{|c|}{ Probable Depression } & \multicolumn{6}{|c|}{ Major Depression } \\
\hline & \multicolumn{2}{|c|}{$\begin{array}{c}\text { No } \\
(n=436)\end{array}$} & \multicolumn{2}{|c|}{$\begin{array}{c}\text { Yes } \\
(n=133)\end{array}$} & \multirow[b]{2}{*}{$\chi^{2}$} & \multirow[b]{2}{*}{$\mathrm{V}$} & \multicolumn{2}{|c|}{$\begin{array}{c}\text { No } \\
(n=540)\end{array}$} & \multicolumn{2}{|c|}{$\begin{array}{c}\text { Yes } \\
(n=29)\end{array}$} & \multirow[b]{2}{*}{$x^{2}$} & \multirow[b]{2}{*}{$\mathrm{V}$} \\
\hline & $n$ & $\%$ & $n$ & $\%$ & & & $n$ & $\%$ & $n$ & $\%$ & & \\
\hline \multicolumn{13}{|l|}{ Parity } \\
\hline Primiparous & 270 & 61.9 & 68 & 51.1 & $\begin{array}{c}4.928 \\
*\end{array}$ & 0.09 & 329 & 60.9 & 9 & 31.0 & $\begin{array}{c}10.197 \\
* *\end{array}$ & 0.13 \\
\hline Multiparous & 166 & 38.1 & 65 & 48.9 & & & 211 & 39.1 & 20 & 69.0 & & \\
\hline \multicolumn{13}{|l|}{ Previous Abortions } \\
\hline No & 336 & 77.1 & 83 & 62.4 & $\underset{* *}{11.280}$ & 0.14 & 398 & 73.7 & 21 & 72.4 & 0.024 & \\
\hline Yes & 100 & 22.9 & 50 & 37.6 & & & 142 & 26.3 & 8 & 27.6 & & \\
\hline \multicolumn{13}{|l|}{ Pregnancy Planning } \\
\hline No & 56 & 12.8 & 24 & 18.0 & 2.282 & & 73 & 13.5 & 7 & 24.1 & 2.569 & \\
\hline Yes & 380 & 87.2 & 109 & 82.0 & & & 467 & 83.5 & 22 & 75.9 & & \\
\hline $\begin{array}{c}\text { Pregnancy Reaction } \\
\text { Positive }\end{array}$ & 418 & 95.9 & 111 & 83.5 & $\underset{* * *}{24.026}$ & 0.21 & 507 & 93.9 & 22 & 75.9 & $\begin{array}{c}13.684 \\
* * *\end{array}$ & 0.16 \\
\hline
\end{tabular}


Table 2. Cont.

\begin{tabular}{|c|c|c|c|c|c|c|c|c|c|c|c|c|}
\hline $\begin{array}{c}\text { Negative } \\
\text { Pregnancy Complication }\end{array}$ & 18 & 4.1 & 22 & 16.5 & & & & 6.1 & 7 & 24.1 & & \\
\hline No & 406 & 93.1 & 117 & 88.0 & \multirow{2}{*}{3.637} & & 496 & 91.9 & 27 & 93.1 & \multirow[t]{2}{*}{0.058} & \\
\hline Yes & 30 & 6.9 & 16 & 12.0 & & & 44 & 8.1 & 2 & 6.9 & & \\
\hline Second Trimester & \multicolumn{2}{|c|}{$\begin{array}{c}\text { No } \\
(n=472)\end{array}$} & \multicolumn{2}{|c|}{$\begin{array}{c}\text { Yes } \\
(n=97)\end{array}$} & & & \multicolumn{2}{|c|}{$\begin{array}{c}\text { No } \\
(n=546)\end{array}$} & \multicolumn{2}{|c|}{$\begin{array}{c}\text { Yes } \\
(n=23)\end{array}$} & & \\
\hline Parity & & & & & & & & & & & & \\
\hline Primiparous & 292 & 61.9 & 46 & 47.4 & $\begin{array}{c}6.959 \\
* *\end{array}$ & 0.11 & 327 & 59.9 & 11 & 47.8 & 1.332 & \\
\hline Multiparous & 180 & 38.1 & 51 & 52.6 & & & 219 & 40.1 & 12 & 52.2 & & \\
\hline \multicolumn{13}{|l|}{ Previous Abortions } \\
\hline No & 348 & 73.7 & 71 & 73.2 & \multirow[t]{2}{*}{0.012} & & 402 & 73.6 & 17 & 73.9 & \multirow[t]{2}{*}{0.001} & \\
\hline Yes & 124 & 26.3 & 26 & 26.8 & & & 144 & 26.4 & 6 & 26.1 & & \\
\hline \multicolumn{13}{|l|}{ Pregnancy Planning } \\
\hline No & 59 & 12.5 & 21 & 21.6 & \multirow{2}{*}{$\begin{array}{l}5.575 \\
*\end{array}$} & \multirow{2}{*}{0.10} & 72 & 13.2 & 8 & 34.8 & \multirow{2}{*}{$\underset{* *}{8.519}$} & \multirow[t]{2}{*}{0.12} \\
\hline Yes & 413 & 87.5 & 76 & 78.4 & & & 474 & 86.8 & 15 & 65.2 & & \\
\hline Pregnancy Reaction & & & & & \multirow{3}{*}{$\begin{array}{c}7.265 \\
* *\end{array}$} & \multirow{3}{*}{0.11} & & & & & \multirow{3}{*}{$\begin{array}{l}7.935 \\
* *\end{array}$} & \multirow{3}{*}{0.12} \\
\hline Positive & 445 & 94.3 & 84 & 86.6 & & & 511 & 93.6 & 18 & 78.3 & & \\
\hline Negative & 27 & 5.7 & 13 & 13.4 & & & 35 & 6.4 & 5 & 21.7 & & \\
\hline \multicolumn{13}{|l|}{ Pregnancy Complication } \\
\hline No & 400 & 84.7 & 76 & 78.4 & \multirow[t]{2}{*}{2.407} & & 460 & 84.2 & 16 & 69.6 & \multirow[t]{2}{*}{3.480} & \\
\hline Yes & 72 & 15.3 & 21 & 21.6 & & & 86 & 15.8 & 7 & 30.4 & & \\
\hline Third Trimester & \multicolumn{2}{|c|}{$\begin{array}{c}\text { No } \\
(n=447)\end{array}$} & \multicolumn{2}{|c|}{$\begin{array}{c}\text { Yes } \\
(n=122)\end{array}$} & & & & 42) & & & & \\
\hline Parity & & & & & & & & & & & & \\
\hline Primiparous & 271 & 60.6 & 67 & 54.9 & 1.295 & & 322 & 59.4 & 16 & 59.3 & 0.001 & \\
\hline Multiparous & 176 & 39.4 & 55 & 45.1 & & & 220 & 40.6 & 11 & 40.7 & & \\
\hline Previous Abortions & & & & & & & & & & & & \\
\hline No & 327 & 73.2 & 92 & 75.4 & 0.251 & & 399 & 73.6 & 20 & 74.1 & 0.003 & \\
\hline Yes & 120 & 26.8 & 30 & 24.6 & & & 146 & 26.4 & 7 & 25.9 & & \\
\hline Pregnancy Planning & & & & & & & & & & & & \\
\hline No & 56 & 12.5 & 24 & 19.7 & $\begin{array}{c}4.048 \\
*\end{array}$ & 0.08 & 72 & 13.3 & 8 & 29.6 & $\begin{array}{c}5.687 \\
*\end{array}$ & 0.10 \\
\hline Yes & 391 & 87.5 & 98 & 80.3 & & & 470 & 86.7 & 19 & 70.4 & & \\
\hline Pregnancy Reaction & & & & & & & & & & & & \\
\hline Positive & 424 & 94.9 & 105 & 86.1 & $\begin{array}{c}11.328 \\
* *\end{array}$ & 0.14 & 508 & 93.7 & 21 & 77.8 & $\begin{array}{c}10.010 \\
* *\end{array}$ & 0.13 \\
\hline Negative & 23 & 5.1 & 17 & 13.9 & & & 34 & 6.3 & 6 & 22.2 & & \\
\hline Pregnancy Complication & & & & & & & & & & & & \\
\hline No & 355 & 79.4 & 86 & 70.5 & $\begin{array}{c}4.380 \\
*\end{array}$ & 0.09 & 427 & 78.8 & 14 & 51.9 & $\begin{array}{c}10.698 \\
* *\end{array}$ & 0.14 \\
\hline Yes & 92 & 20.6 & 36 & 29.5 & & & 115 & 21.2 & 13 & 48.1 & *** & \\
\hline Attendance at Maternal & & & & & & & & & & & & \\
\hline Classes & & & & & 10.357 & & & & & & 6.190 & \\
\hline No & 149 & 33.3 & 60 & 49.2 & $* *$ & 0.14 & 193 & 35.6 & 16 & 59.3 & * & 0.10 \\
\hline Yes & 298 & 66.7 & 62 & 50.8 & & & 349 & 64.4 & 11 & 40.7 & & \\
\hline
\end{tabular}

$$
{ }^{*} p<0.05,{ }^{* *} p<0.01,{ }^{* * *} p<0.001 \text {. }
$$

Table 3. Characteristics of women with probable depression and major depression regarding psychological variables.

\begin{tabular}{|c|c|c|c|c|c|c|c|c|c|c|c|c|}
\hline \multirow{3}{*}{ First Trimester } & \multicolumn{6}{|c|}{ Probable Depression } & \multicolumn{6}{|c|}{ Major Depression } \\
\hline & \multicolumn{2}{|c|}{$\begin{array}{c}\text { No } \\
(n=436)\end{array}$} & \multicolumn{2}{|c|}{$\begin{array}{c}\text { Yes } \\
(n=133)\end{array}$} & \multirow[b]{2}{*}{$\chi^{2}$} & \multirow[b]{2}{*}{ V } & \multicolumn{2}{|c|}{$\begin{array}{c}\text { No } \\
(n=540)\end{array}$} & \multicolumn{2}{|c|}{$\begin{array}{c}\text { Yes } \\
(n=29)\end{array}$} & \multirow[b]{2}{*}{$\chi^{2}$} & \multirow[b]{2}{*}{$\mathrm{V}$} \\
\hline & $n$ & $\%$ & $n$ & $\%$ & & & $n$ & $\%$ & $n$ & $\%$ & & \\
\hline $\begin{array}{c}\text { History of Depression } \\
\text { No } \\
\text { Yes }\end{array}$ & $\begin{array}{c}412 \\
24\end{array}$ & $\begin{array}{c}94.5 \\
5.5\end{array}$ & $\begin{array}{c}115 \\
18\end{array}$ & $\begin{array}{l}86.5 \\
13.5\end{array}$ & $\begin{array}{c}9.610 \\
* *\end{array}$ & 0.13 & $\begin{array}{c}506 \\
34\end{array}$ & $\begin{array}{c}93.7 \\
6.3\end{array}$ & $\begin{array}{c}21 \\
8\end{array}$ & $\begin{array}{l}72.4 \\
27.6\end{array}$ & $\underset{* * *}{18.247}$ & 0.19 \\
\hline $\begin{array}{l}\text { Worsening Mood in Previous } \\
\text { Pregnancies }(n=234) \\
\text { No } \\
\text { Yes }\end{array}$ & $\begin{array}{c}147 \\
21\end{array}$ & $\begin{array}{l}87.5 \\
12.5\end{array}$ & $\begin{array}{l}50 \\
16\end{array}$ & $\begin{array}{l}75.8 \\
24.4\end{array}$ & $\begin{array}{c}4.908 \\
*\end{array}$ & 0.15 & $\begin{array}{c}184 \\
30\end{array}$ & $\begin{array}{l}86.0 \\
14.0\end{array}$ & $\begin{array}{c}13 \\
7\end{array}$ & $\begin{array}{l}65.0 \\
35.0\end{array}$ & $\begin{array}{c}6.049 \\
*\end{array}$ & 0.16 \\
\hline
\end{tabular}


Table 3. Cont.

\begin{tabular}{|c|c|c|c|c|c|c|c|c|c|c|c|c|}
\hline $\begin{array}{l}\text { Trait Anxiety } \\
\text { No } \\
\text { Yes }\end{array}$ & $\begin{array}{c}428 \\
8\end{array}$ & $\begin{array}{c}98.2 \\
1.8\end{array}$ & $\begin{array}{c}109 \\
24\end{array}$ & $\begin{array}{l}82.0 \\
18.0\end{array}$ & $\begin{array}{c}50.455 \\
* * *\end{array}$ & 0.30 & $\begin{array}{c}491 \\
49\end{array}$ & $\begin{array}{c}90.9 \\
9.1\end{array}$ & $\begin{array}{c}21 \\
8\end{array}$ & $\begin{array}{l}72.4 \\
27.6\end{array}$ & $\underset{* * *}{10.463}$ & 0.14 \\
\hline $\begin{array}{l}\text { State Anxiety } \\
\text { No } \\
\text { Yes }\end{array}$ & $\begin{array}{c}419 \\
17\end{array}$ & $\begin{array}{c}96.1 \\
3.9\end{array}$ & $\begin{array}{l}93 \\
40\end{array}$ & $\begin{array}{l}69.9 \\
30.1\end{array}$ & $\underset{* * *}{77.467}$ & 0.37 & $\begin{array}{c}513 \\
27\end{array}$ & $\begin{array}{c}95.0 \\
5.0\end{array}$ & $\begin{array}{c}24 \\
5\end{array}$ & $\begin{array}{l}82.8 \\
17.2\end{array}$ & $\begin{array}{c}7.770 \\
* *\end{array}$ & 0.12 \\
\hline Second Trimester & & & & & & & & & & & & \\
\hline $\begin{array}{c}\text { History of Depression } \\
\text { No } \\
\text { Yes }\end{array}$ & $\begin{array}{c}446 \\
26\end{array}$ & $\begin{array}{c}94.5 \\
5.5\end{array}$ & $\begin{array}{l}81 \\
16\end{array}$ & $\begin{array}{l}83.5 \\
16.5\end{array}$ & $\underset{* * *}{14.206}$ & 0.16 & $\begin{array}{c}510 \\
36\end{array}$ & $\begin{array}{c}93.4 \\
6.6\end{array}$ & $\begin{array}{c}17 \\
6\end{array}$ & $\begin{array}{l}73.9 \\
26.1\end{array}$ & $\underset{* * *}{12.267}$ & 0.15 \\
\hline $\begin{array}{c}\text { Worsening Mood in Previous } \\
\text { Pregnancies }(n=234) \\
\text { No } \\
\text { Yes }\end{array}$ & $\begin{array}{c}163 \\
20\end{array}$ & $\begin{array}{l}89.1 \\
10.9\end{array}$ & $\begin{array}{l}34 \\
17\end{array}$ & $\begin{array}{l}66.7 \\
33.3\end{array}$ & $\begin{array}{c}15.040 \\
* * *\end{array}$ & 0.25 & $\begin{array}{c}191 \\
31\end{array}$ & $\begin{array}{l}86.0 \\
14.0\end{array}$ & $\begin{array}{l}6 \\
6\end{array}$ & $\begin{array}{l}50.0 \\
50.0\end{array}$ & $\underset{* *}{11.106}$ & 0.22 \\
\hline $\begin{array}{c}\text { Trait Anxiety } \\
\text { No } \\
\text { Yes }\end{array}$ & $\begin{array}{c}459 \\
13\end{array}$ & $\begin{array}{c}97.2 \\
2.8\end{array}$ & $\begin{array}{l}78 \\
19\end{array}$ & $\begin{array}{l}80.4 \\
19.6\end{array}$ & $\begin{array}{c}42.958 \\
* * *\end{array}$ & 0.28 & $\begin{array}{c}522 \\
24\end{array}$ & $\begin{array}{c}95.6 \\
4.4\end{array}$ & $\begin{array}{c}15 \\
8\end{array}$ & $\begin{array}{l}65.2 \\
34.8\end{array}$ & $\underset{* * *}{38.396}$ & 0.26 \\
\hline $\begin{array}{l}\text { State Anxiety } \\
\text { No } \\
\text { Yes }\end{array}$ & $\begin{array}{c}464 \\
8\end{array}$ & $\begin{array}{c}98.3 \\
1.7\end{array}$ & $\begin{array}{l}75 \\
22\end{array}$ & $\begin{array}{l}77.3 \\
22.7\end{array}$ & $\underset{* * *}{70.950}$ & 0.35 & $\begin{array}{c}523 \\
23\end{array}$ & $\begin{array}{c}95.8 \\
4.2\end{array}$ & $\begin{array}{c}16 \\
7\end{array}$ & $\begin{array}{l}69.6 \\
30.4\end{array}$ & $\underset{* * *}{30.385}$ & 0.23 \\
\hline Third Trimester & $(n=$ & & $(n=$ & & & & & & & & & \\
\hline $\begin{array}{c}\text { History of Depression } \\
\text { No } \\
\text { Yes }\end{array}$ & $\begin{array}{c}423 \\
24\end{array}$ & $\begin{array}{c}94.6 \\
5.4\end{array}$ & $\begin{array}{c}104 \\
18\end{array}$ & $\begin{array}{l}85.2 \\
14.8\end{array}$ & $\underset{* * *}{12.348}$ & 0.15 & $\begin{array}{c}507 \\
35\end{array}$ & $\begin{array}{c}93.5 \\
6.5\end{array}$ & $\begin{array}{c}20 \\
7\end{array}$ & $\begin{array}{l}74.1 \\
25.9\end{array}$ & $\underset{* * *}{14.259}$ & 0.16 \\
\hline $\begin{array}{c}\text { Worsening Mood in Previous } \\
\text { Pregnancies }(n=234) \\
\text { No } \\
\text { Yes }\end{array}$ & $\begin{array}{c}155 \\
22\end{array}$ & $\begin{array}{l}87.6 \\
12.4\end{array}$ & $\begin{array}{l}42 \\
15\end{array}$ & $\begin{array}{l}73.7 \\
26.3\end{array}$ & $\begin{array}{c}5.246 \\
*\end{array}$ & 0.16 & $\begin{array}{c}191 \\
32\end{array}$ & $\begin{array}{l}85.7 \\
14.3\end{array}$ & $\begin{array}{l}6 \\
5\end{array}$ & $\begin{array}{l}54.5 \\
45.5\end{array}$ & $\begin{array}{c}7.619 \\
*\end{array}$ & 0.18 \\
\hline $\begin{array}{c}\text { Trait Anxiety } \\
\text { No } \\
\text { Yes }\end{array}$ & $\begin{array}{c}436 \\
11\end{array}$ & $\begin{array}{c}97.5 \\
2.5\end{array}$ & $\begin{array}{c}101 \\
21\end{array}$ & $\begin{array}{l}82.8 \\
17.2\end{array}$ & $\begin{array}{c}39.298 \\
* * *\end{array}$ & 0.26 & $\begin{array}{c}519 \\
23\end{array}$ & $\begin{array}{c}95.8 \\
4.2\end{array}$ & $\begin{array}{c}18 \\
9\end{array}$ & $\begin{array}{l}66.7 \\
33.3\end{array}$ & $\underset{* * *}{41.005}$ & 0.27 \\
\hline $\begin{array}{c}\text { State Anxiety } \\
\text { No } \\
\text { Yes }\end{array}$ & $\begin{array}{c}438 \\
9\end{array}$ & $\begin{array}{c}98.0 \\
2.0\end{array}$ & $\begin{array}{l}90 \\
32\end{array}$ & $\begin{array}{l}73.8 \\
26.2\end{array}$ & $\underset{* * *}{84.056}$ & 0.38 & $\begin{array}{c}510 \\
32\end{array}$ & $\begin{array}{c}94.1 \\
5.9\end{array}$ & $\begin{array}{c}18 \\
9\end{array}$ & $\begin{array}{l}66.7 \\
33.3\end{array}$ & $\underset{* * *}{28.939}$ & 0.23 \\
\hline
\end{tabular}

\subsection{Predictors of Depression in Each Trimester}

Predictors of the presence of probable depression and major depression in each of the trimesters are shown in Tables 4 and 5.

Age, previous miscarriages, state anxiety, and perceived stress were predictors of probable depression in the first trimester. Specifically, being aged 30 years old or younger $(\mathrm{OR}=2.55)$, having had previous miscarriages $(\mathrm{OR}=3.28)$, having a high state of anxiety $(\mathrm{OR}=3.97)$ as well as higher perceived stress $(\mathrm{OR}=1.24)$ increased the likelihood of probable depression in the first trimester.

In the second trimester, having had probable depression in the first trimester $(\mathrm{OR}=13.61)$ as well as higher perceived stress in the first $(\mathrm{OR}=1.14)$ and second trimesters $(\mathrm{OR}=1.35)$ increased the likelihood of having probable depression.

In the third trimester, the predictors of probable depression were having had major depression in the first trimester $(\mathrm{OR}=5.43)$, probable depression in the second trimester $(\mathrm{OR}=6.19)$, and an elevated state of anxiety $(\mathrm{OR}=4.88)$ and higher perceived stress $(\mathrm{OR}=1.16)$ in the third trimester.

In the first trimester, the predictors of major depression were having perceived that pregnancy had a negative influence on employment $(\mathrm{OR}=3.20)$ and having higher per- 
ceived stress $(\mathrm{OR}=1.13)$. In the second trimester, the predictors were being aged 30 years old or younger $(\mathrm{OR}=1.26)$ and experiencing higher perceived stress $(\mathrm{OR}=1.27)$. In the third trimester, the predictors were being aged 30 years old or younger $(\mathrm{OR}=7.23)$ and experiencing higher perceived stress in the second $(\mathrm{OR}=1.16)$ and in the third trimesters $(\mathrm{OR}=1.18)$.

Table 4. Predictors of probable depression in each trimester.

\begin{tabular}{|c|c|c|c|c|}
\hline Predictors in the First Trimester & B & WALD & $p$ & OR $[95 \%$ IC] \\
\hline Age $\leq 30$ years & 0.94 & 4,04 & 0.045 & $2.55[1.02-6.37]$ \\
\hline Previous Miscarriages & 1.19 & 7.13 & 0.008 & $3.28[1.37-7.85]$ \\
\hline High State Anxiety & 1.38 & 4.70 & 0.03 & $3.97[1.14-13,81]$ \\
\hline High Perceived Stress & 0.22 & 24.70 & $<0.001$ & $1.24[1.14-1.35]$ \\
\hline Constant & -6.49 & 38.88 & $<0.001$ & 0.002 \\
\hline Cox and Snell R2 & & & 0.343 & \\
\hline Nagelkerke's R2 & & & 0.501 & \\
\hline \multicolumn{5}{|l|}{ Predictors in the Second Trimester } \\
\hline Probable Depression in the 1st Trimester & 2.61 & 21.41 & $<0.001$ & $13.61[4.51-41.14]$ \\
\hline Higher Perceived Stress in 1st Trimester & 0.12 & 6.53 & 0.011 & $1.14[1.03-1.24]$ \\
\hline Higher Perceived Stress in 2nd Trimester & 0.30 & 26.46 & $<0.001$ & $1.35[1.21-1.52]$ \\
\hline Constant & -5.90 & 29.98 & $<0.001$ & 0.003 \\
\hline Cox and Snell R2 & & & 0.402 & \\
\hline Nagelkerke's R2 & & & 0.611 & \\
\hline \multicolumn{5}{|l|}{ Predictors in the Third Trimester } \\
\hline Major Depression in the 1st Trimester & 1.69 & 6.49 & 0.011 & 5.43 [1.48-19.95] \\
\hline Probable Depression in the 2nd Trimester & 1.82 & 16.47 & $<0.001$ & $6.19[2.47-14.91]$ \\
\hline High State Anxiety in 3rd Trimester & 1.59 & 4.49 & 0.034 & $4.88[1.13-21.13]$ \\
\hline Higher Perceived Stress in 3rd Trimester & 0.15 & 15.57 & $<0.001$ & $1.16[1.08-1.25]$ \\
\hline Constant & -5.22 & 36.82 & $<0.001$ & 0.005 \\
\hline Cox and Snell R2 & & & 0.368 & \\
\hline Nagelkerke's R2 & & & 0.555 & \\
\hline
\end{tabular}

Table 5. Predictors of major depression in each trimester.

\begin{tabular}{|c|c|c|c|c|}
\hline Predictors in the First Trimester & B & WALD & $p$ & OR $[95 \% \mathrm{IC}]$ \\
\hline Negative influence of Pregnancy on Work Situation & 1.16 & 5.31 & 0.021 & $3.20[1.19-8.63]$ \\
\hline High Perceived Stress & 0.12 & 12.21 & $<0.001$ & $1.13[1.05-1.21]$ \\
\hline Constant & -5.45 & 37.28 & $<0.001$ & 0.004 \\
\hline Cox and Snell R2 & & & 0.094 & \\
\hline Nagelkerke's R2 & & & 0.210 & \\
\hline \multicolumn{5}{|l|}{ Predictors in the Second Trimester } \\
\hline Age $\leq 30$ years & 1.71 & 5.33 & $<0.001$ & $1.26[1.13-23.42]$ \\
\hline High Perceived Stress in the 2nd Trimester & 0.24 & 15.66 & $<0.001$ & $1.27[1.13-1.42]$ \\
\hline Constant & -9.13 & 27.10 & $<0.001$ & 0.000 \\
\hline Cox and Snell R2 & & & 0.127 & \\
\hline Nagelkerke's R2 & & & 0.398 & \\
\hline \multicolumn{5}{|l|}{ Predictors in the Third Trimester } \\
\hline Age $\leq 30$ years & 1.98 & 4.98 & 0.026 & $7.23[1.27-41.08]$ \\
\hline High Perceived Stress in the 2nd Trimester & 0.15 & 4.22 & 0.04 & $1.16[1.01-1.34]$ \\
\hline High Perceived Stress in 3rd Trimester & 0.16 & 4.20 & 0.04 & $1.18[1.01-1.37]$ \\
\hline Constant & -11.51 & 20.60 & $<0.001$ & 0.000 \\
\hline Cox and Snell R2 & & & 0.147 & \\
\hline Nagelkerke's R2 & & & 0.485 & \\
\hline
\end{tabular}




\section{Discussion}

\subsection{Trajectory of Depression during Pregnancy}

The aim of the present longitudinal study was to analyse the trajectory of both probable depression and major depression during pregnancy. The prevalence of probable depression ranged from $17.0 \%$ to $23.4 \%$, and the prevalence of major depression ranged from $4.0 \%$ to $5.1 \%$. During pregnancy, the variation in probable depression and major depression followed the same pattern or trajectory. In both cases, the prevalence was the highest in the first trimester, decreased in the second trimester, and increased again in the third trimester, although not reaching the rates observed in the first trimester. It is possible that, as stated by Rallis et al. [14], this trajectory of depression may occur because the beginning of a pregnancy can be a time of strong psychological vulnerability involving multiple factors, such as hormonal, physical, psychological, and emotional adjustment to the new situation of pregnancy, which can increase vulnerability to the development of depressive symptoms. On the other hand, the third trimester is another critical time, as it also involves major physical and emotional changes in view of the approaching birth.

The findings of the few studies that have assessed the presence of depressive symptomatology in all three trimesters of pregnancy are variable. The same pattern as the one observed in the present study was also observed in studies conducted in China [16], Turkey [15], and Australia [14]. However, in the study by Weng et al. [17] conducted in China, although the two most prevalent times of depressive symptomatology were also the first and third trimester, the prevalence was higher in the latter than in the former. On the other hand, the prevalence observed in a study in Korea [20] follows a rising pattern from the first to the third trimester, and Ajinkya et al. [19] found to have the highest prevalence of depressive symptomatology in the second trimester and the lowest values in the third trimester. However, it should be noted that these studies use different scales and cut-off points to assess probable depression, such as the EPDS [17], the BDI [15,19], and the SDS [16]. On the other hand, some studies have identified different latent trajectory groups based on the total scores of depressive symptoms [43,44].

Regarding the trajectory of major depression, we can only compare our data with those reported by Marchesi et al. [18] in Italy, as this is the only study including a longitudinal follow-up in the three trimesters of gestation. The trajectory reported is similar to that observed in the present study, following a V-shaped pattern, with the prevalence of depression being the highest in the first and third trimesters, although in this case, the prevalence was the highest in the third trimester.

The differences between the trajectory observed in the present study and those observed in other studies may be due to cultural differences, the quality of prenatal care received and the levels of satisfaction with this prenatal care, the professionals carrying out prenatal care (midwives and/or obstetricians), health conditions, and the type and accessibility of healthcare (public/private) in each country.

\subsection{Variables Associated with Probable Depression and Major Depression in Pregnancy}

The second objective of this study was to identify the sociodemographic, pregnancyrelated, and psychological variables associated with both probable depression and major depression in each trimester. This will enable us to determine whether any variables are specifically associated with a particular trimester of pregnancy, with one condition of depression or the other, or with both equally.

Regarding the sociodemographic variables, both probable depression and major depression were associated with younger age. In addition, an age less than or equal to 30 years old was found to be a predictor of major depression in the second and third trimester. This relationship was also found in other research in which being younger than 25 [31], younger than 20 [22], or aged 15-20 [25] was associated with an increased risk of antenatal depression. This relationship can be explained by the fact that younger women tend to have a more unfavourable and unstable economic position and lower-paid jobs, leading to lower income level [25]. 
On the other hand, in all trimesters of pregnancy, both probable depression and major depression were associated with lower educational level. These findings are consistent with those of previous studies $[15,22,23,25]$. This relationship can be explained by the fact that a low level of education is often related to socio-economic disadvantage [23], which is one of the most worrying aspects for women when facing motherhood, as they may fear that they will not be able to meet their children's needs [45]. Low socio-economic status is also often accompanied by increased stress, which is, in turn, considered a risk factor for depression. In the present study, lower income was associated with the presence of probable depression in the first and second trimesters. This finding is consistent with the findings of other authors, who observed associations with low socioeconomic status [22,24] and family income below the minimum wage [27]. Employment status is one of the factors related to both educational and economic status. Being unemployed was associated with the presence of probable depression in the first trimester and with major depression in the second and third trimesters, as was also found in other studies [22,30,31]. Giardinelli et al. [30] suggested that this relationship may be due to the fact that not working implies having a smaller social support network and some degree of isolation. Likewise, not being in paid work is associated with lower educational attainment and lower economic resources. Another possible factor explaining this association is the frustration that the women had after observing that being pregnant prevented them from having equal access to the labour market, as some women reported being dismissed from work, not being able to apply for positions of responsibility, or being obliged to request reduced working hours or job adaptations that often did not correspond to their professional category. This hypothesis would explain the observed association between the perception of the women that pregnancy had had a negative influence on their work situation and the presence of probable depression at all stages of pregnancy and with major depression in the first and second trimesters. It was also a predictor of major depression in the first trimester. Of note is a sociodemographic variable with which no association was found at any time during pregnancy with either probable or major depression. Not having a partner has been associated in some studies with antenatal depression $[17,23,29]$, but not in this research. However, only 29 women claimed to not have a partner in the present study, and this could influence the results. On the other hand, the explanation for this finding could be that what is associated with depression is not so much with having or not having a partner, but with the quality of the relationship and how it is perceived by the woman as satisfactory or unsatisfactory.

Regarding pregnancy-related variables, parity, specifically being multiparous, was associated with probable depression in the first and second trimesters as well as with having major depression in the first trimester, and in this case, it was a predictor variable. This finding may be explained by the fact that women with more children bear a greater physical and emotional burden due to the demands of caring for a larger number of family members. While some studies corroborate this association [19,23,26,32], Redinger et al. [36] found the opposite, i.e., being nulliparous was associated with gestational depression.

Having had previous miscarriages was a predictor of probable depression in the first trimester, and no relationship was found at any other time or with the presence of major depression. One possible explanation is that miscarriages often occur in the first trimester and, therefore, those women with a history of miscarriage may experience greater distress at this time because of the fear of a repeat of the situation and because, in turn, they are reminded of the previous experience. This situation could make them vulnerable to developing depressive symptoms. Once this trimester is over, these symptoms disappear. Although other authors have also found an association between previous miscarriages and antenatal depression $[19,29,32]$, in another study [31], this relationship was not significant.

Unplanned pregnancy was associated in the second and third trimesters with both probable depression and major depression. This association has been widely documented $[19,29,30,32,46]$. There are several possible explanations for this. First, unplanned or unwanted pregnancies carry an enormous emotional burden [47]. Another 
explanation involves the socio-demographic characteristics of women who find themselves in a situation of unwanted pregnancy. As such, women may not be financially or socially prepared to cope with the demands of pregnancy [48]. These women also tend to have more unstable social environments and may feel a lack of security and support from their partner (if any) and have more marital conflict $[49,50]$. All of these circumstances increase the risk of antenatal depression. On the other hand, having had a negative reaction to the confirmation of pregnancy was associated with both probable depression and major depression in all three trimesters. Although this variable may be related to the previous variable (unplanned/wanted pregnancy), negative reactions do not always occur in this context. However, the explanation for this relationship may be similar, as negative reactions such as resignation and anger, among others, are an added emotional burden. Moreover, the woman may feel guilty for having such reactions, even if the pregnancy was wanted, as the idealisation of motherhood and social pressure impose that it is politically incorrect to say (or think) something negative at this time.

With regard to the presence of pregnancy complications, an association was found with both probable depression and major depression in the third trimester. Other authors $[19,25]$ have also found this relationship. The presence of complications is a stressful life event and a psychological burden for women that favours the appearance of depressive symptomatology. It should be borne in mind that what women consider to be a complication may not be a complication from a clinical point of view. For example, some women perceive back pain or nausea as a complication, whereas for a health professional, both entities would be considered physiological or normal. Therefore, the important point in psychological terms is not what is reflected in the medical history, but the woman's perception of the process.

Not attending maternal education classes was associated with both probable depression and major depression in the third trimester, the time of pregnancy when the women were assessed, as this is when these classes begin. Maternal education sessions aim to equip the mother and her partner with knowledge and skills to prepare them to cope with physical, emotional, and lifestyle changes in those areas of which they feel the most insecure. The mere fact of being in contact with other women in the same situation and with the same needs as well as having a healthcare professional who listens empathetically and resolves doubts can minimize the impact of the possible worries that most women have in pregnancy.

In terms of psychological variables, in all three trimesters, probable depression and major depression were both associated with a history of depression, the perceived worsening of mood in previous pregnancies, and with the presence of trait anxiety, state anxiety, and higher perceived stress.

The relationship with the presence of a history of depression has also been found by other authors in cohorts of pregnant women [23,31] as well as at specific times during pregnancy $[17,26,46]$. The explanation for this relationship is the existence of a vulnerability to depression that may be intensified by changes brought about by pregnancy and motherhood (e.g., sleep, rest, altered body image). Another explanation is that many women who are undergoing pharmacological treatment for a depressive episode may choose to interrupt it when they become aware of the pregnancy for fear of possible teratogenic effects on the foetus [51] and, therefore, the symptoms will possibly worsen and be prolonged throughout the perinatal period. In relation to this, in the present study, multiparous women who perceived a worsening of mood in previous pregnancies were more likely to have probable depression and major depression.

State anxiety was associated with both probable and major depression in all three trimesters of pregnancy and was also a predictor of probable depression in the first and third trimesters. This relationship is widely documented $[26,33,35,36]$. The main explanation is the frequent comorbidity of both disorders [35,52]. Ross et al. [53] concluded that more than $50.0 \%$ of pregnant women with depression had also been diagnosed with anxiety. 
In terms of perceived stress, our data indicate that a high level of perceived stress is a predictor of both entities of depression at all points in pregnancy. This association is frequently found with other measures of stress such as the presence of stressful life events $[33,36,46]$, and it must be borne in mind that pregnancy is considered stressful for many women because of the changes it brings about in their lives [54].

Thus, the study findings indicate that all of the psychological variables considered were associated with both probable depression and major depression at all stages of pregnancy. Therefore, it is crucial to take these variables into account in routine follow-up examinations.

Interpretation of the results obtained in the present research should take into consideration the limitation that no pre-pregnancy baseline measurements of psychological variables are available. Therefore, it is not possible to determine if there were any changes in pregnancy relative to the usual condition of the participants. The strengths of the study are mainly methodological. In particular, it is a longitudinal study (the first in Spain), which enabled us to identify both the associations between the variables and the predictors of depression status. In addition, the data were collected prospectively and individually by administering the questionnaires face-to-face to a large sample at all assessment times in order to minimize recall bias. This, in turn, resulted in low sample attrition. This is also the only study in which the prevalence and variables associated with both depressive symptomatology (assessed with a screening instrument) and major depression (assessed by clinical interview) have been analyzed together, providing a more complete view of the subject. This enabled us to understand the trajectory of two very different situations in terms of the severity of depression and to examine the similarities between the associated variables. In general, although the results do not indicate any notable differences, as the variables associated with both probable depression and major depression are similar, there are some exceptions. Specifically, previously having had a miscarriage was only associated with probable depression in the first trimester of pregnancy, while suffering pregnancy complications was only associated with major depression in the third trimester. Likewise, the level of income was only associated with probable depression in the first and second trimesters. However, lower educational level, having had a negative reaction to the confirmation of pregnancy, and all of the psychological variables were associated with both types of depression in all three trimesters. This finding reinforces the fact that the EPDS is a valid instrument for detecting women at risk of clinical depression if it is routinely implemented in pregnancy monitoring.

Regarding the clinical implications, this study enabled us to identify the variables associated with the presence of depression at different times. While some of these variables (i.e., sociodemographic variables) are unalterable, others (i.e., psychological variables) are potentially modifiable. Influencing these variables at an early stage could lead to a reduction in the prevalence and adverse consequences of depression. The study findings also enabled us to determine which variables should be assessed routinely in order to enable the necessary help to be offered at each stage, to predict which women are at the greatest risk of developing depression in pregnancy, and to develop specific interventions at the most appropriate time to prevent depression from extending to the postpartum period. The findings could thus help to improve care during the perinatal stage and prevent or reduce the likelihood of the women experiencing depression.

\section{Conclusions}

In the present study, the prevalence of probable depression in pregnancy was $23.4 \%$ in the first trimester, $17.0 \%$ in the second trimester, and $21.4 \%$ in the third trimester. The prevalence of major depression was $5.1 \%$ in the first trimester, $4.0 \%$ in the second, and $4.7 \%$ in the third trimester. The trajectories of both probable depression and major depression throughout pregnancy are therefore the same, being more prevalent in the first (in particular) and third trimesters. Regarding the variables associated with both probable depression and major depression, with the exception of variables such as having suffered 
previous miscarriages and pregnancy complications and/or having a lower level of income, no significant differences were found between probable and major depression. Regarding socio-demographic variables, a younger age, lower level of education, being unemployed, and the perception that pregnancy had a negative influence on employment stands out. Regarding the pregnancy-related variables, not having planned the pregnancy, negative reaction to the confirmation of the pregnancy, and not attending maternal education classes stands out. It should be noted that psychological variables were associated with probable depression and major depression in all three trimesters. In fact, the predictor variables are also mainly psychological, with perceived stress being a predictor for both conditions in all trimesters. The data indicate the importance of integrating mental health care as a part of routine pregnancy follow-up protocols. This would involve conducting assessments throughout pregnancy as early as the first trimester to detect women at risk of suffering depression.

Author Contributions: Conceptualization, M.C.M.; formal analysis, M.B.V.; investigation, M.B.V.; methodology, M.C.M. and M.B.V.; supervision, M.C.M.; data curation, M.C.M. and M.B.V.; writingoriginal draft, M.B.V.; writing - review and editing, M.C.M. and M.B.V. All authors have read and agreed to the published version of the manuscript.

Funding: This research received no external funding.

Institutional Review Board Statement: The study was conducted according to the guidelines of the Declaration of Helsinki, and approved by the Clinical Research Ethics Committee of Galicia (protocol code 2015/437 and date of approval 22 July 2015).

Informed Consent Statement: Informed consent was obtained from all subjects involved in the study.

Data Availability Statement: The data presented in the current study are available from the authors upon request.

Acknowledgments: We would like to especially show our gratitude to the pregnant women who participated for their patience and generous collaboration in this study.

Conflicts of Interest: The authors declare no conflict of interest.

\section{References}

1. WHO. Depresión. Datos y cifras [Depression. Data and numbers]. Available online: https://www.who.int/es/news-room/factsheets/detail/depression (accessed on 5 April 2019).

2. Kamysheva, E.; Skouteris, H.; Wertheim, E.H.; Paxton, S.J;; Milgrom, J. A prospective investigation of the relationships among sleep quality, physical symptoms, and depressive symptoms during pregnancy. J. Affect. Disord. 2010, 123, 317-320. [CrossRef]

3. Bowen, A.; Muhajarine, N. Antenatal depression. Can. Nurse 2006, 102, 26-30.

4. Lee, A.M.; Lam, S.K.; Sze Mun Lau, S.M.; Chong, C.S.; Chui, H.W.; Fong, D.Y. Prevalence, course, and risk factors for antenatal anxiety and depression. Obstet. Gynecol. 2007, 110, 1102-1112. [CrossRef]

5. Míguez, M.C.; Fernández, V.; Pereira, B. Depresión postparto y factores asociados en mujeres con embarazos de riesgo [Postpartum depression and associated factors in women with at-risk pregnancies]. Behav. Psychol. 2017, 25, 47-64.

6. Yonkers, K.A.; Smith, M.V.; Gotman, N.; Belanger, K. Typical somatic symptoms of pregnancy and their impact on a diagnosis of major depressive disorder. Gen. Hosp. Psychiatry 2009, 31, 327-333. [CrossRef] [PubMed]

7. Saeed, A.; Raana, T.; Saeed, A.M.; Humayun, A. Effect of antenatal depression on maternal dietary intake and neonatal outcome: A prospective cohort. Nutr. J. 2016, 15, 64-93. [CrossRef] [PubMed]

8. Eastwood, J.; Ogbo, F.A.; Hendry, A.; Noble, J.; Page, A. The impact of antenatal depression on perinatal outcomes in australian women. PLoS ONE 2017, 12, e0169907. [CrossRef] [PubMed]

9. Goedhart, G.; Snijders, A.C.; Hesselink, A.E.; Van Poppel, N.; Bonsel, G.J.; Vrijkotte, T.G.M. Maternal depressive symptoms in relation to perinatal mortality and morbidity: Results from a large multiethnic cohort study. Psychosom. Med. 2010, 72, 769-776. [CrossRef]

10. Herrero, T.; Driebe, A.; Fratto, V.; Hamlin, A.; Lacoursiere, D.Y.; Ramos, G.A. Positive antenatal Edinburgh Depression Scale: Examining behavioral and pharmacological therapy on maternal and neonatal outcomes. J. Matern. Fetal Neonatal Med. 2018, 1-5. [CrossRef] [PubMed]

11. Felice, E.; Saliba, J.; Grech, V.; Cox, J. Validation of the Maltese version of the Edinburgh Postnatal Depression Scale. Arch. Womens Ment. Health 2006, 9, 75-80. [CrossRef]

12. Matthey, S.; Ross-Hamid, C. Repeat testing on the Edinburgh Depression Scale and the HADS-A in pregnancy: Differentiating between transient and enduring distress. J. Affect. Disord. 2012, 141, 213-221. [CrossRef] 
13. Vázquez, M.B.; Míguez, M.C. Validation of the Edinburgh postnatal depression scale as a screening tool for depression in Spanish pregnant women. J. Affect. Disord. 2019, 246, 515-521. [CrossRef]

14. Rallis, S.; Skouteris, H.; McCabe, M.; Milgrom, J. A prospective examination of depression, anxiety and stress throughout pregnancy. Women Birth 2014, 27, e36-e42. [CrossRef]

15. Yanikkerem, E.; Ay, S.; Mutlu, S.; Goker, A. Antenatal depression: Prevalence and risk factors in a hospital based Turkish sample. J. Pak. Med. Assoc. 2013, 63, 472-477. [PubMed]

16. Yu, Y.; Li, M.; Pu, L.; Wang, S.; Wu, J.; Ruan, L.; Jiang, S.; Wang, Z.; Jiang, W. Sleep was associated with depression and anxiety status during pregnancy: A prospective longitudinal study. Arch. Womens Ment. Health 2017, 20, 695-701. [CrossRef] [PubMed]

17. Weng, S.C.; Huang, J.P.; Huang, Y.L.; Lee, T.S.; Chen, Y.H. Effects of tobacco exposure on perinatal suicidal ideation, depression, and anxiety. BMC Public Health 2016, 16, 623. [CrossRef] [PubMed]

18. Marchesi, C.; Bertoni, S.; Maggini, C. Major and minor depression in pregnancy. Obstet. Gynecol. 2009, 113, 1292-1298. [CrossRef]

19. Ajinkya, S.; Jadhav, P.R.; Srivastava, N.N. Depression during pregnancy: Prevalence and obstetric risk factors among pregnant women attending a tertiary care hospital in Navi Mumbai. Ind. Psychiatry J. 2013, 22, 37-40. [CrossRef]

20. Park, J.H.; Karmaus, W.; Zhang, H. Prevalence of and risk factors for depressive symptoms in Korean women throughout pregnancy and in postpartum period. Asian Nurs. Res. 2015, 9, 219-225. [CrossRef]

21. Míguez, M.C.; Vázquez, M.B. Risk factors for antenatal depression: A review. World J. Psychiatr. 2021, in press.

22. Bödecs, T.; Szilagyi, E.; Cholnoky, P.; Sándor, J.; Gonda, X.; Rihmer, Z.; Horváth, B. Prevalence and psychosocial background of anxiety and depression emerging during the first trimester of pregnancy: Data from a Hungarian population-based sample. Psychiatr. Danub. 2013, 25, 352-358.

23. De Vargas, C.; Coll, N.; Da Silveira, M.; Garcia-Bassani, D.; Netsi, E.; César Wehrmeister, F.; ... Stein, A. Antenatal depressive symptoms among pregnant women: Evidence from a Southern Brazilian population-based cohort study. J. Affect. Disord. 2017, 209, 140-146. [CrossRef]

24. Husain, N.; Parveen, A.; Husain, M.; Saeed, Q.; Jafri, F.; Rahman, R.; Tomenson, B.; Chaudhry, I.B. Prevalence and psychosocial correlates of perinatal depression: A cohort study from urban Pakistan. Arch. Womens Ment. Health 2011, 14, 395-403. [CrossRef]

25. Thompson, O.; Ajayi, I. Prevalence of antenatal depression and associated risk factors among pregnant women attending antenatal clinics in Abeokuta north local government area, Nigeria. Depress. Res. Treat. 2016, 16, 1-15. [CrossRef]

26. Banti, S.; Mauri, M.; Oppo, A.; Borri, C.; Rambelli, C.; Ramacciotti, D.; Montagnani, M.S.; Camilleri, V.; Cortopassi, S.; Rucci, P.; et al. From the third month of pregnancy to 1 year postpartum. Prevalence, incidence, recurrence, and new onset of depression. Results from the perinatal depression-research \& screening unit study. Compr. Psychiatry 2011, 52, 343-351. [CrossRef] [PubMed]

27. Benute, G.R.G.; Nomura, R.M.Y.; Reis, J.S.; Fraguas-Junior, R.; Lucia, M.C.S.; Zugaib, M. Depression during pregnancy in women with a medical disorder: Risk factors and perinatal outcomes. Clinics 2011, 65, 1127-1131. [CrossRef] [PubMed]

28. Ogbo, F.A.; Eastwood, J.; Hendry, A.; Jalaludin, B.; Agho, K.E.; Barnett, B.; Page, A. Determinants of antenatal depression and postnatal depression in Australia. BMC Psychiatry 2018, 18, 49. [CrossRef] [PubMed]

29. Weobong, B.; Asbroek, A.H.A.; Soremekun, S.; Manu, A.A.; Owusu-Agyei, S.; Prince, M.; Kirkwood, B.R. Association of antenatal depression with adverse consequences for the mother and newborn in rural Ghana: Findings from the DON population-based cohort study. PLoS ONE 2014, 9, e116333. [CrossRef]

30. Giardinelli, L.; Innocenti, A.; Benni, L.; Stefanini, M.C.; Lino, G.; Lunardi, C.; Svelto, V.; Afshar, S.; Bovani, R.; Castellini, G.; et al. Depression and anxiety in perinatal period: Prevalence and risk factors in an Italian sample. Arch. Womens Ment. Health 2012, 15, 21-30. [CrossRef]

31. Kheirabadi, G.R.; Maracy, M.R. Perinatal depression in a cohort study on Iranian women. J. Res. Med. Sci. 2010, 15, 41-49.

32. González-Mesa, E.; Kabukcuoglu, K.; Körükcü, O.; Blasco, M.; Ibrahim, N.; Kavas, T. Cultural factors influencing antenatal depression: A cross-sectional study in a cohort of Turkish and Spanish women at the beginning of the pregnancy. J. Affect. Disord. 2018, 238, 256-260. [CrossRef]

33. Mohammad, K.I.; Gamble, J.; Creedy, D.K. Prevalence and factors associated with the development of antenatal and postnatal depression among Jordanian women. Midwifery 2011, 27, 238-245. [CrossRef]

34. Waldie, K.E.; Peterson, E.R.; D'Souza, S.; Underwood, L.; Pryor, J.E.; Carr, P.A.; Grant, C.; Morton, S.M.B. Depression symptoms during pregnancy: Evidence from Growing Up in New Zealand. J. Affect. Disord. 2015, 186, 66-73. [CrossRef] [PubMed]

35. Fadzil, A.; Balakrishnan, K.; Razali, R.; Sidi, H.; Malapan, T.; Japaraj, R.P.; Midin, M.; Jaafar, N.R.N.; Das, S.; Manaf, M.R.A. Risk factors for depression and anxiety among pregnant women in Hospital Tuanku Bainun, Ipoh, Malaysia. Asia Pac. Psychiatry 2013, 5, 7-13. [CrossRef] [PubMed]

36. Redinger, S.; Norris, S.A.; Pearson, R.M.; Richter, L.; Rochat, T. First trimester antenatal depression and anxiety: Prevalence and associated factors in an urban population in Soweto, South Africa. J. Dev. Orig. Health Dis. 2018, 9, 30-40. [CrossRef]

37. Rich-Edwards, J.; Kleinman, K.; Abrams, A.; Harlow, B.L.; McLaughlin, T.J.; Joffe, H.; Gillman, M.W. Sociodemographic predictors of antenatal and postpartum depressive symptoms among women in a medical group practice. J. Epidemiol. Community Health 2006, 60, 221-227. [CrossRef]

38. Cox, J.; Holden, J.M.; Sagovsky, R. Detection of postnatal depression. Development of the 10-item Edinburgh Postnatal Depression Scale. Br. J. Psychiatry 1987, 150, 782-786. [CrossRef] [PubMed] 
39. First, M.B.; Spitzer, R.L.; Gibbon, M.; Williams, J.B.W. Entrevista clínica estructurada para los trastornos del eje I del DSM-IVversión clínica (SCID-CV) [Structured Clinical Interview for DSM-IV Axis I Disorders, Clinician Version (SCID-CV)]. 1997. Versión Clínica Ed. Masson. Barcelona. Available online: https:/ / libreriaolejnik.cl/fichas/90090.pdf (accessed on 17 November 2015).

40. Remor, E. Psychometric properties of a European Spanish version of the Perceived Stress Scale (PSS). Span. J. Psychol. 2006, 9 , 86-93. [CrossRef] [PubMed]

41. Cohen, S.; Kamarck, T.; Mermelstein, R. A global measure of perceived stress. J. Health Soc. Behav. 1983, 24, 385-396. [CrossRef]

42. Spielberg, C.D.; Gorsuch, R.L.; Lushene, R.E. Manual of State-Trait Anxiety Inventory; Consulting Psichologists Press: Palo Alto, CA, USA, 1970.

43. Ahmed, A.; Bowen, A.; Feng, C.X.; Muhajarine, N. Trajectories of maternal depressive and anxiety symptoms from pregnancy to five years postpartum and their prenatal predictors. BMC Pregnancy Childbirth 2019, 19, 26. [CrossRef]

44. Lee, H.; Kim, K.E.; Kim, M.Y.; Park, C.G.; Han, J.Y.; Choi, E.J. Trajectories of depressive symptoms and anxiety during pregnancy and associations with pregnancy stress. Int. J. Environ. Res. Public Health 2021, 18, 2733. [CrossRef] [PubMed]

45. Segre, L.S.; O'Hara, M.W.; Arndt, S.; Stuart, S. The prevalence of postpartum depression: The relative significance of three social status indices. Soc. Psychiatry Psychiatr. Epidemiol. 2007, 42, 316-321. [CrossRef] [PubMed]

46. Marcos-Nájera, R.; Rodríguez-Muñoz, M.F.; Lara, M.A.; Navarrete, L.; Le, H.N. A cross-cultural analysis of the prevalence and risk factors for prenatal depression in Spain and Mexico. Cult. Med. Psychiatry 2020, 1-14. [CrossRef]

47. Koutra, K.; Vassilaki, M.; Georgiou, V.; Koutis, A.; Bitsios, P.; Chatzi, L.; Kogevinas, M. Antenatal maternal mental health as determinant of postpartum depression in a population based mother-child cohort (Rhea Study) in Crete, Greece. Soc. Psychiatry Psychiatr. Epidemiol. 2014, 49, 711-721. [CrossRef] [PubMed]

48. Sedgh, G.; Bankole, A.; Oye-Adeniran, B.; Adewole, I.F.; Singh, S.; Hussain, R. Unwanted pregnancy and associated factors among Nigerian women. Int. Fam. Plan Perspect. 2006, 32, 175-184. [CrossRef]

49. Bouchard, G. Adult couples facing a planned or an unplanned pregnancy. J. Fam. Issues 2005, 26, 619-637. [CrossRef]

50. Bouchard, G.; Boudreau, J.; Hébert, R. Transition to parenthood and conjugal life: Comparisons between planned and unplanned pregnancies. J. Fam. Issues 2006, 27, 1512-1531. [CrossRef]

51. Vesga-López, O.; Blanco, C.; Keyes, K.; Olfson, M.; Grant, B.F.; Hasin, D.S. Psychiatric disorders in pregnant and postpartum women in the United States. Arch. Gen. Psychiatry 2008, 65, 805. [CrossRef]

52. Falah-Hassani, K.; Shiri, R.; Dennis, C.L. Prevalence and risk factors for comorbid postpartum depressive symptomatology and anxiety. J. Affect. Disord. 2016, 198, 142-147. [CrossRef]

53. Ross, L.E.; Gilbert Evans, S.E.; Sellers, E.M.; Romach, M.K. Measurement issues in postpartum depression part 1: Anxiety as a feature of postpartum depression. Arch. Womens Ment. Health 2003, 6, 51-57. [CrossRef]

54. Van den Bergh, B.R.H.; Mulder, E.J.H.; Mennes, M.; Glover, V. Antenatal maternal anxiety and stress and the neurobehavioural development of the fetus and child: Links and possible mechanisms. A review. Neurosci. Biobehav. Rev. 2005, 29, 237-258. [CrossRef] [PubMed] 